\section{Einstellung zur Integration im Zusammenhang mit sozialer Inklusion - Eine Fragebogenerhebung in österreichischen Integrations- und Regelschulklassen}

\section{Susanne Schwab \\ Universität Graz}

Da in Österreich, wie auch in vielen anderen Ländern, immer mehr Schülerlinnen mit sonderpädagogischem Förderbedarf (SPF) in integrativen Regelklassen (sog. Integrationsklassen) unterrichtet werden, nimmt auch das Forschungsinteresse diesbezüglich deutlich zu. Während immer noch mangelnde Klarheit in Bezug auf die verwendeten Begrifflichkeiten wie z. B. Integration vs. Inklusion vorherrscht, ergeben sich in Hinblick auf die Effekte der Integration vorwiegend zwei Forschungsfragen. Die erste beschäftigt sich mit den Auswirkungen, welche Integration auf die Schulleistungen der integrierten Schüler/innen, aber auch ihrer Mitschüler/innen hat. Die zweite hinterfragt die soziale Integration und das Wohlbefinden der Schülerlinnen. Mögliche Vergleiche bei diesen Erhebungen werden zwischen folgenden Gruppen gezogen: Zwischen Schüler/innen mit SPF, welche in Integrations- und Sonderschulklassen unterrichtet werden, zwischen Schüler/innen mit und ohne SPF in denselben Schulklassen, aber auch zwischen Schülerlinnen ohne SPF in Schulklassen, in denen sich integrierte Schülerlinnen befinden, und zwischen Regelschulklassen (für eine Übersicht siehe z.B. Schwab 2014). Worin sich die Forschung grundsätzlich einig zu sein scheint, ist, dass eine gelingende Inklusion eine entsprechend positive Einstellung der beteiligten Personen wie beispielsweise der Lehrerlinnen (z.B. de Boer u.a. 2011), aber auch der Bevölkerung an sich (z.B. Schwab u.a. 2014) voraussetzt. Insbesondere zur Einstellung von Lehrerlinnen finden sich sowohl zahlreiche nationale als auch internationale Studienergebnisse. Für Österreich gab es jedoch bislang keine größere quantitative Untersuchung, bei der die Einstellung von Schüler/innen mit und ohne SPF im Vordergrund stand. Folglich wurde die Längsschnittstudie ATIS-SI (Attitudes Towards Inclusion of Students with disabilities related to Social Inclusion) konzipiert, um die Einstellungen zur schulischen Integration von Schülerlinnen sowie die soziale Partizipation zu untersuchen.

\section{Ziele der ATIS-SI Studie}

Im Rahmen der ATIS-SI Studie ist es insbesondere von Interesse, ob sich die Einstellung von Schülerlinnen aus Integrations- und Regelklassen unterscheiden und welchen Einfluss die Art der Behinderung des zu integrierenden Kindes auf die Einstellung hat. Nebst Fragen zur Einstellung werden auch solche zur sozialen Partizipation sowie zur Klasse und zum Unterricht gestellt. Im Längsschnitt sollen förderliche und auch hinderliche Bedingungen für eine positive Einstellung und soziale Inklusion identifiziert werden.

\section{Überblick über die verwendeten Messinstrumente}

Um die Einstellung gegenüber Peers mit Behinderung zu erfassen, wurde eine Kurzversion der Chedoke-McMaster Attitudes towards Children with Handicaps Skala (CATCH; Rosenbaum u.a. 1986) verwendet, wobei die Items jeweils für vier verschiedene Fallbeschreibungen (Kind mit Körperbehinderung, Kind mit Lernbehinderung, Kind mit geistiger Behinderung sowie Kind mit Verhaltensauffälligkeit) vorgegeben wurden. Die soziale Partizipation wurde zum einen aus Sicht der Lehrerlinnen mittels Social Participation Questionnaire (SPQ; Koster u.a. 2008) erfasst, wobei diese Items aus Fragen zur Peerakzeptanz, zu Freundschaften, Interaktionen und zur Selbstsicht der Schüler/innen bestanden. Die Schüler/innen selbst wurden gebeten, ihre fünf besten Freund/innen zu nennen und anzugeben, wie viel ihrer Pausenzeit sie mit ihren Mitschüler/innen verbringen. Zudem wurden einzelne Skalen aus dem Fragebogen zur Erfassung emotionaler und sozialer Schulerfahrungen von Grundschulkindern dritter und vierter Klassen (FEESS 3-4; Rauer/Schuck 2003) vorgegeben. Hierzu zählten sowohl Soziale Integration, Schullust, Klassenklima, Gefühl des Angenommenseins durch die Lehrperson, Einsamkeit (4 Items der Illinois Loneliness and Social Satisfaction Scale von Asher u.a. 1984) als auch das soziale Selbstkonzept (Social Description Questionnaire II-Short von Marsh u.a. 2005) sowie die Intention, die Schule zu verlassen (siehe dazu Pijl u. a. 2014). Verhaltensstärken und -probleme wurden mit dem Strengths and Difficulties Questionnaires (SDQ; Goodman 1997) erfasst und normabweichendes Verhalten im Unterricht zusätzlich 
mit einer selbstkonstruierten Skala von Eckstein und Reusser (in Vorbereitung) abgefragt. Auch kooperatives Lernen (Schwab/Helm, in Vorbereitung) und individualisierter Unterricht (Gebhardt u.a. 2014) wurden erhoben.

\section{Durchführung und Überblick über die Teilnehmerlinnen}

Insgesamt haben 1'115 Schülerlinnen (36,6\% der 4. und $63,4 \%$ der 7 . Schulstufe) aus 63 Schulklassen aus der Steiermark, Niederösterreich und dem Burgenland im Herbst 2013 am Projekt teilgenommen. Mehr als $11 \%$ der Stichprobe wies einen SPF auf. Etwas über die Hälfte der Teilnehmer/innen besuchte eine Integrationsklasse, in der mindestens ein/e Schüler/in mit SPF gemeinsam in der Klasse unterrichtet wurde. Im Sommer 2014 fand die zweite Messung statt, an der 61 Schulklassen teilnahmen.

\section{Erste Ergebnisse der Erhebung im Herbst 2013}

Bezugnehmend auf die Einstellung zu Kindern mit Behinderung zeigte sich für den ersten Messzeitpunkt, dass diese eher neutral zu sehen ist. Dabei ist jene gegenüber Kindern mit Körperbehinderung oder Lernbehinderung etwas positiver als jene gegenüber Kindern mit geistiger Behinderung oder Verhaltensauffälligkeit. Einflüsse auf Individual- (Geschlecht, SPF vs. kein SPF) oder Klassenebene (Schulstufe, Integrations- vs. Regelklasse) konnten nur für das Geschlecht gefunden werden. Diesbezüglich wiesen Mädchen eine positivere Einstellung auf.

Bei den Lehrkrafteinschätzungen wurde klar ersichtlich, dass die soziale Partizipation für Schülerlinnen mit SPF niedriger eingeschätzt wurde als für Schülerlinnen ohne SPF. Die Schülerlinnenbefragung ergab ein ähnliches Bild. In Bezug auf die Einsamkeit zeigte sich, dass sich etwa $15 \%$ der Schülerlinnen mit SPF extrem einsam fühlten, während es bei jenen ohne SPF immerhin auch noch etwa $5 \%$ waren.
Etwas erfreulicher waren die Ergebnisse zum schulischen Wohlbefinden, worin sich Schüler/innen mit und ohne SPF nicht signifikant unterschieden. Mädchen wiesen ein höheres schulisches Wohlbefinden auf als Jungen.

\section{Schlussfolgerungen und Vorausschau}

Wenngleich noch wenige Analysen durchgeführt wurden, so lassen die Ergebnisse insgesamt dennoch darauf schließen, dass es wichtig ist, sowohl förderliche als auch hinderliche Bedingungen für schulische Inklusion im Auge zu behalten. Im gemeinsamen Unterricht von Schüler/innen mit und ohne SPF fühlen sich Schüler/innen mit SPF oft einsam und verfügen über eine geringere soziale Partizipation als ihre Peers. Insofern wäre es vonnöten, künftig mehr Ressourcen in die Förderung der sozialen Partizipation von Schülerlinnen mit SPF in Integrationsklassen zu investieren. Auch was die Einstellung von Schülerlinnen gegenüber ihren Peers mit Behinderung betrifft, zeigt sich Handlungsbedarf. Es gilt, im Zuge der längsschnittlichen Erhebung, aber auch unter Zuhilfenahme von qualitativen Befragungen herauszufinden, welche Variablen eine positive und welche eine negative Einstellung hervorrufen und wie diese in Zukunft beeinflusst werden können. Damit schulische Inklusion gut umgesetzt werden kann, bedarf es einer laufenden Begleitung durch die Forschung, um Einflussvariablen zu identifizieren und Interventionsprogramme entwickeln zu können. Ziel ist es, sowohl die akademische als auch die soziale Entwicklung aller Schüler/innen maximal zu fördern. Dies wurde von Österreich auch durch die Ratifizierung der UN-Konvention über die Rechte von Menschen mit Behinderung bestätigt (UN Convention 2006, § 24, 2 e; siehe UN General Assembly 2006). Insofern gilt es, die Thematik weiter zu verfolgen, um dem Ziel einer inklusiven Gesellschaft näher zu kommen.

Weitere Informationen und Literaturangaben können eingeholt werden bei susanne.schwab@ uni-graz.at 